\title{
Künftige Tendenzen von Internet und Internet Governance
}

\subsection{Generelle Trends und grundlegende Perspektiven}

In den letzten drei Jahrzehnten hat sich das Internet - wie nur von wenigen prognostiziert - von einem experimentellen Netzwerk von Forschungsinstitutionen in den USA zu einer globalen technischen Infrastruktur entwickelt, die nahezu alle Aktivitäten in Handel und Wirtschaft, Finanzen und Verkehr, Politik und Verwaltung, Kultur, Kommunikation, Medien und Kunst tangiert, bestreitet und transformiert - und zwar auch ohne dass dies jeweils spezifisch geplant worden wäre. Vielmehr wurden diese Entwicklungen durch unendlich viele und unterschiedliche Faktoren und Akteure vorangetrieben. Dies dürfte auch weiterhin so von statten gehen - gleich ob sich schon manche Prozesse in Bezug auf das Internet abzeichnen, die meisten aber noch ihrer Entfaltung harren (DIE ZEIT, Nr. 9, 21. Februar 2013, S. 30).

Die unübersichtliche Komplexität des Internets beschränkt - über die ohnehin prinzipielle Unsicherheit sozialer Prognosen hinaus - die Validität von Voraussagen in Bezug auf die künftigen Entwicklungstendenzen. Allenfalls Schätzungen sind möglich, die gleichwohl in den jeweils gängigen Geschäftsmodellen und Marketingstrategien mit möglichst attraktiven und vielversprechenden Überschriften versehen wurden - man denke nur an das zufällige Label Web 2.0! - und sicherlich auch künftig werden: So zirkulieren derzeit neben Web 3.0 bzw. SemanticWeb noch BigData (der große Datenhaufen) und Internet of things. Allerdings mutieren sie unversehens zu sich selbst erfüllenden Prophezeiungen, da die Entwicklung des Internets und seiner Anwendungsoptionen nicht nur Produkte, sondern im 
Zusammenwirken mit anderen Megatrends oder „Metaprozessen“ (Krotz 2007, 27) gewissermaßen in dialektischer Wechselwirkung zugleich Faktoren und Motoren dieser Entwicklung sind. Dadurch verkomplizieren sich künftige Tendenzen zusätzlich, denn Trends firmieren gleichermaßen als Folgen wie als Antriebe (Tenscher und Vierig 2010, 8ff; Hepp 2011, 48ff; Kübler 2012a). Dies sei an folgenden Megatrends erläutert:

1. Globalisierung ist sicherlich der am häufigsten angesprochene, wohl auch grundlegendste und einschneidendste globale Trend, ohne dass er hier über die üblichen Verweise auf die anhaltende Zunahme, Verdichtung und Integration weltweiter Wirtschaftsbeziehungen hinaus im Detail dargestellt werden soll und kann (Giddens 1996; Beck 1997; Deutscher Bundestag 2002; Hepp 2004, Varwick 2011). Ohne leistungsfähige, schnelle, interaktive Netze zum Austausch von Daten, Informationen und Bildern, also ohne wachsende Konnektivität (Hepp 2006), wäre Globalisierung nicht nur kaum denkbar, sondern erst recht nicht entwicklungsfähig. Denn die Netze eröffnen, beschleunigen und generieren selbst Globalisierungsprozesse, weil mit digitalisierten Daten überall - möglichst an verfügbaren und kostengünstigen Standorten - produziert, gehandelt und abgerechnet werden kann. Viele Wertschöpfungskomponenten (in Entwicklung, Produktion und Distribution), vor allem im nahezu überall zunehmenden Anteil des Dienstleistungssektors am Sozialprodukt zirkulieren ständig rund um den Globus.

2. Technische Voraussetzungen für Computer und Internet sind bekanntlich die Digitalisierung, d.h. die Transformation aller analogen, physischen Daten in binäre Codes, und damit die Entgegenständlichung und Virtualisierung der codierten, gespeicherten ,Welten'. Mit den anhaltend wachsenden Speicher- und Steuerungskapazitäten der Computer und Netze sind diese Prozesse längst noch nicht beendet, sondern erfassen weitere und noch komplexere Gegenstandsfelder, die vor allem in Produktion und Dienstleistung, in Verkehr und Finanzen, Kultur und Medien ständig neue Optionen ermöglichen und damit weitere Automatisierungsprozesse antreiben. Dadurch können bislang nur physische

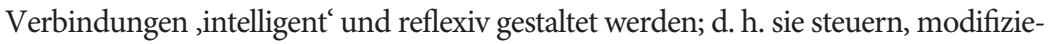
ren und optimieren sich selbst: etwa Netze für die Energieversorgung, Produktion, Distribution und Konsumtion, für Finanzen, soziale Beobachtung und Beratung, für medizinische Versorgung, Handel und Konsum. Allerdings steigen auch ihre Risiken bei Ausfall, Missbrauch, Störung oder bei willentlicher Manipulation, Spionage und Schädigung.

3. All diese Prozesse sind ohne ökonomische Konsequenzen nicht möglich; sie binden Kapital und generieren auch Gewinne. Entsprechende Zusammenhänge 
werden allerdings unter verschiedenen Vorzeichen und mit wechselnden Paradigmen analysiert: Pauschal lassen sie sich als wachsende Ökonomisierung oder Kommerzialisierung beschreiben, weil immer mehr Segmente gesellschaftlichen Lebens in den kapitalistischen Verwertungsprozess einbezogen werden: etwa die medizinische Versorgung und Pflege, die Partnersuche und Familie, Kultur und Freizeit, soziale Beziehungen, Bildung und Kunst. Auch die Umwandlung von öffentlicher Daseinsvorsorge in private Dienstleistungen rechnet zu diesen Veränderungen, die auch von der notorischen Knappheit öffentlicher Haushaltsmittel angetrieben wird. Aus der Perspektive der technischen Vermittlung wird von fortschreitende Mediatisierung oder Medialisierung (Krotz 2007; Meyen 2009) gesprochen, weil zunehmend ehedem direkte, persönliche Kommunikationsprozesse durch Medien bewerkstelligt, aber auch verändert und vervielfältigt werden, so dass die Medienoptionen nicht nur neue, meist lukrative Märkte generieren, sondern auch die moderne Gesellschaft als ganze prägen. Gängig ist dafür die Kennzeichnung als Mediengesellschaft geworden (Saxer 2012; Kübler 2009, 27f), ob und wie berechtigt sie auch ist. Mediale Kommunikation rekurriert zu maßgeblichen Anteilen auf kommerzieller Werbung, das mächtigste und wirkungsvollste Ferment von Medialisierung, denn sie finanziert großenteils den expandierenden Medienmarkt, ja sie ist konstitutive Basis für ihn, wie sie umgekehrt die Medien nach ihren Strategien ausprägt und ständig neue Mediengenres und -formate kreiert. Als primärer wie auch als sekundärer Verwertungsmarkt verbindet sie alle anderen Marktsektoren, beschleunigt und potenziert unablässig die Warenzirkulation.

4. In der Wirtschaft selbst, in der so genannten realen wie in der sie flankierenden Finanzwirtschaft, erzeugen Globalisierung und Wettbewerb Tendenzen der weiteren wirtschaftlichen Konzentration, betriebsintern solche der anhaltenden Verdichtung, Beschleunigung und Komplexitätsexpansion. Sie verlangen jeweils höheren logistischen, Steuerungs- und Kontrollaufwand, der nur durch weitere Vernetzung, Technisierung, Informatisierung und Automatisierung bewältigt werden kann. Außerdem motiviert die stete Sättigung neuer Märkte die Entwicklung ständig neuer, noch speziellerer Dienstleistungen, um die Märkte weiter $\mathrm{zu}$ differenzieren und $\mathrm{zu}$ fragmentieren. All diese Erfordernisse werden inzwischen mit dem mysteriösen Begriff BigData umschrieben - in den 1980er Jahren knüpfte man schon ähnliche Erwartungen an die so genannten „künstliche Intelligenz" (Dreyfus 1985; Michie und Johnston 1985), die womöglich bald wieder eine Renaissance erleben wird (Russell und Novrig 2012). Umrissen werden mit BigData noch recht vage die zu entwickelnden Kapazitäten sämtlicher Wirtschaftszweige, die ständig anfallenden und wachsenden Datenmassen - und zwar jedweden Formats und Mediums - gezielt, rational und effizient zu sortieren, 
zu selegieren und zu klassifizieren, dass sie für die jeweils gesteckten Ziele, Zwecke und Problemlösungen vollständig, transparent, aber auch erfolgversprechend eingesetzt werden können. Künftige Entwicklungen scheinen sich mithin auch hier zu differenzieren: Nicht mehr die unbedachte Sammlung und Speicherung von riesigen Datenmengen ist vorrangig gefragt, sondern ihre Filterung und Aufbereitung zur Verfügbarkeit für je konkrete Aufgaben. Allenfalls Bruchteile der vorhandenen Daten seien - so jüngste Schätzungen - dafür nötig, vor allem aber ihre speziellen Verknüpfungen und Interpretationen, so dass die zu bewältigenden Zwecke sicher, schnell und wirksam vonstatten gehen können. Zahlreiche große Konzerne - in Deutschland beispielweise die Allianz-Versicherung, die Drogeriemarkt-Kette $d m$ und der Automobilherstelle BMW-, wagen sich inzwischen an BigData-Projekte, aber ihre Erfolgsaussichten sind noch recht unsicher, viele der Investitionen daher riskant (Fischermann und Hamann 2011).

5. Zwei weitere Entwicklungen flankieren und verstärken die beschriebenen Trends: zum einen die fortschreitende Mobilisierung, zum anderen die anhaltende Personalisierung und Intimisierung der Kunden bzw. Verbraucher. Mit der Einführung und Verbreitung flächendeckender digitaler Mobilfunknetze (D-Netz Ende 1980er und Anfang 1990er Jahre in Deutschland, Österreich und der Schweiz) - vulgo: Handy - begann die bis heute anhaltende Mobilisierung medialer Kommunikation, also ihre Ablösung von stationären Geräten durch Smartphones, IPads und Tablet PCs und die permanente Verfügbarkeit digitaler Information bzw. die ebensolche Erreichbarkeit ihrer Nutzer. Sie korrespondieren mit den schon umschriebenen Prozessen der Globalisierung und Medialisierung, wie sie umgekehrt diese weiter befördern und beschleunigen. Arbeiten und Kommunizieren an jedem Ort, zu jeder Zeit, möglichst mit der ganzen Welt, ungeachtet von Kulturen und Traditionen, Reisen überall hin dies kennzeichnet den globalisierten, mediatisierten Weltbürger, dem territoriale und personale Bezüge entschwinden (Meckel 2011; Schirrmacher 2013): Physische, soziale und emotionale „Deterritoralität“ (Hepp 2011, 103ff) wird kompensiert durch Vernetzung und ,connectivity' (Hepp 2006), fokussiert auf die gerade benutzte Plattform, den jeweiligen Kommunikationszweck und die jeweils nützlichen Partner. Dadurch verwandeln sich sicherlich menschlichen Beziehungen und Interaktionen, sie werden in jedem Fall pragmatischer, eindeutiger, weniger risikoreich, aber sie verlieren auch wichtige soziale und emotionale Komponenten. Inzwischen kursieren schon etliche Warnungen über die Folgen der digitalen Beziehung(slosigkeit)en und der unentwegten Erreichbarkeit, wie berechtigt sie auch immer sind (Gaschke 2009; Meckel 2011; Pauer 2012; Schirrmacher 2013). 
6. Mit der Masse von kommunikativen Optionen, Dienstleistungen und Daten wird die präzise Vermessung der Adressaten und Verbraucher immer wichtiger und für Absatz wie Adressierungen dringlicher. Die verfügbaren Daten ermöglichen zudem seine Profilierung bis in seine intimsten Eigenschaften hinein. Mehr und mehr produzieren dafür automatische Programme - so genannte Filter Bubbles (Pariser 2012) - exakte Angaben, ergänzen und modifizieren sie selbstständig und treten so mit dem Kunden in fiktive Dialoge, so dass er sich in einer vermeintlichen Face-to-faceSituation wähnt. Sie schafft Vertrauen und motiviert so eher zum Kauf und - auf Dauer - zur Konsumgewohnheit. Wenn aus allgemeiner soziologischer Perspektive für die (post)modernen Gesellschaften ein anhaltender Trend zur Individualisierung des bzw. der Einzelnen, d. h. zu seiner bzw. ihrer zunehmenden Herauslösung aus traditionellen Vergemeinschaftungsformen wie Klasse, Sippe und Familie und zur komplementären Bildung funktionaler Gruppen in Beruf, Ausbildung, Nachbarschaft, politischer und kultureller Betätigung diagnostiziert wird (Beck 1986, 205ff), dann korrespondiert mit dieser neuen Art der - wie auch immer begründeten - Vergesellschaftung die zweckrationale, kommerzielle Personalisierung durch Medien, Werbung und Dienstleistung: Sie hofiert und umgarnt das wirtschaftlich verwertbare Ich ungleich eindringlicher und vielversprechender, suggeriert ihm Pseudo-Individualität durch Waren und mediales Charisma und entschädigt ihn zumindest symbolisch für etliche reale soziale Entbehrungen: „Erzähle Dein ganzes Leben“, warb Facebook im Februar 2012 bei der Einführung des neuen Formats Facebook-Chronik - und stelle es aus, hätte noch dazugehört, so dass es alle kennen und wohl auch (be)nutzen können.

Weitere Geräte werden den Usern buchstäblich auf oder gar unter die Haut rücken: Experimentiert wird mit Uhren, die alle individuellen Daten - auch Blutdruck, Puls, chronische Medikamenteneinnahme und Untersuchungsergebnisse etc. - registrieren und vorhalten. In Bruchteilen könnten Kliniken, Ärzte, Versicherungen, Polizei und andere Stellen die wichtigen, womöglich lebensrettenden Daten abrufen und mit ihren Systemen vergleichen; aber auch Missbräuche und illegale Anwendungen könnten dabei wohl nicht vermieden werden (Koller 2012). Solchen noch äußerlichen und kontrollierbaren Geräten könnten später subkutane Chips folgen, die unter der Haut platziert werden. Für manche Handicaps wie Hörschäden, Sehverlust und Blindheit oder auch Störungen der Bewegungsabläufe infolge von Krankheit könnten solche künstlichen, digitalen Steuerungshilfen sicherlich willkommen und nützlich sein; doch auch diese digitalen Krücken sind nicht gefeit vor Zweckentfremdung und Manipulation. 
Mit diesen Entwicklungsskizzen sind sicherlich nicht alle grundlegenden Trends (post)moderner Gesellschaften umrissen; allein die hier aufgeführten signalisieren jedenfalls, wie komplex und kontingent, nämlich abhängig von einer unübersehbaren Vielfalt von Faktoren und Zusammenhängen, sie ausfallen (können) und wie schwierig bis unmöglich Übersichten oder gar Gewichtungen sind, weshalb J. Habermas (1985) schon frühzeitig die strukturelle "Unübersichtlichkeit" als essentielles Kennzeichen der (post)moderne Gesellschaft gekennzeichnet hat. Auch bleibt zu beachten, dass sich solcher Wandel in den Regionen der Erde, erst recht infolge ihres Entwicklungsstandes und ihres Transformationspotentiale ganz unterschiedlich herausstellt; die gängige Einteilung in (entwickelte) Industriestaaten, Schwellen- und Entwicklungsländer ist nur ein recht schlichter Versuch der groben Typisierung. Über globale Entwicklungstrends lässt sich mithin wenig Seriöses und Verlässliches prognostizieren; auch die selbstsichersten Entwürfe (Bolz 2001; Beck 2007) stellen sich meist schnell als kurzatmige und komplexitätsreduzierende Momentaufnahme heraus.

\subsection{Disziplinäre Perspektiven}

Ungeachtet der Identifizierung und auch der Konstruktion solcher Megatrends werden die künftigen Entwicklungen des Internets auch aus konventionellen disziplinären Blickwinkeln vorgenommen: also als technische, ökonomische, soziale, politische, kulturell-kommunikative und als noch etliche andere Dynamiken wobei diese nicht gänzlich isoliert werden können, sondern vielfach verzahnt sind. Auch dafür sind alle möglichen Modelle und Ableitungen denkbar: von einigermaßen seriösen, aber wenig spektakulären Fortschreibungen von Trends über einigermassen methodisch kontrollierte Szenarien bis hin zu kühnen, visionären Entwürfen.

Aus ökonomischer Sicht geht es primär um weitere Verwertungsoptionen des Internets und die Schaffung neuer Märkte von Medien, Werbung, personaler und gewerblicher Kommunikation. Sie werden realisiert durch die Entwicklung und den Verkauf jeweils neuer Geräte (hardware) und Programme (software). Dabei kommt es nicht nur zur Kreation neuer Produkte, Genres und Formate, vielmehr finden auch Wettbewerbs- und Verdrängungsprozesse zwischen, alten', meist weniger technisierten und komplexen, ,neuen' Versionen statt, wie es sich gegenwärtig für Print- und alle physischen Produkte (z. B. Spiel) beobachten lässt. Jeweils müssen neue Geschäftsmodelle konzipiert werden, weshalb die meisten internationalen Kommunikations- und Medienkonzerne crossmedial operieren 
und ihre Kommunikationsofferten wie Inhalte multicodal und multipel vermarkten können.

Die sekundäre Vermarktung erweist sich als weitere Verdichtung, Expansion und Effektivierung sämtlicher Informations- und Datenprozesse in allen Branchen, Produktions- und Dienstleistungssegmenten. Dazu müssen digitale Technologien weiter in Produkte und Transaktionen integriert werden und dort möglichst automatisch wie reibungslos funktionieren: also in Kühlschränke und Küchen, in Autos und Logistik, in Laborexperimenten, Produktionsabläufen und im Zahlungsverkehr. Netze und Systeme müssen dafür leistungsfähiger, stabiler, aber auch flexibler, geeigneter für vielerlei Anwendungen, auch möglichst störungsfrei werden. Dies sind Herausforderungen und Aufgaben für die Technik, jedoch auch für die Politik und die private Gefahrenabwehr.

In technischer Hinsicht müssen die Systeme und Netze ständig optimiert werden, den jeweiligen An- und Verwendungen gewachsen sein, weiter dezentralisiert, differenziert und spezifiziert, aber auch gesichert und robust werden. Diese Anforderungen werden unter den genannten Schlagworten diskutiert: BigData als neue Strategien der optimierten Datenauswertung, Web 3.0 oder Semantic Web als wachsende Fähigkeit der Netze, sich den Aufgabenanforderungen durch Bedeutungs- und Bilderkennung anzupassen, gewissermaßen zu lernen, ,intelligenter ${ }^{\prime} \mathrm{zu}$ werden, weitgehend automatisch weitere Komponenten zu erschließen und selbsttätig zu bewältigen, „cloud computing“ als zunehmende Auslagerung von Infrastrukturen, Plattformen, Programmen und Daten in flottierende Netzwerke, um einerseits die Mobilität und Flexibilität zu erhöhen und um andererseits die Verarbeitungskapazität stark zu erhöhen. Mögliche Risiken wie die enorme Anfälligkeit solcher Netzressourcen durch Spionage, Hacking und Manipulation, können nur erahnt werden (Fischermann und Hamann 2011; 2011a). Optimierungen sind auch die bereits begonnene Umstellung auf ein neues Internet-Protokoll, nämlich auf IPv6 (ohne dass die User etwas merken sollen), die kontinuierliche Steigerung der Rechnerkapazitäten bis zum Hundertfachen des heute Möglichen, genannt Exaflop-Rechner, sowie die ebenfalls ständige Expansion der Netzkapazitäten durch Komprimierung, aber auch Erweiterung. Konzipiert sind auch die Dezentralisierung und Aufteilung des Internets nach der Qualität und Sensibilität der Daten, ihrer Sicherheit und nach möglichen Nutzerkreisen.

Wie in den Kapiteln vorab ausgeführt, stellen sich die politischen Fragen nicht minder vielschichtig. Weitgehend verflogen sind vorerst die ursprünglichen Visionen, mit dem Internet und mit den es tragenden zivilgesellschaftlichen Gruppierungen ließen sich gewissermaßen schleichend neue, kollaborative Strukturen in der Welt insgesamt durchsetzen, da das Netz weder Territorien 
und Grenzen noch Regime und institutionelle Machtverhältnisse kennt oder braucht (Eisel 2011). Inzwischen behauptet sich wieder eine stärker national ausgerichtete Netzpolitik. Damit werden Netz-Regulierungen wieder von der Beschaffenheit des jeweiligen politischen Regimes und seines Selbstverständnisses abhängig und weniger von den speziellen Erfordernissen des Netzes und seiner User. Wenn sich politische Verfasstheiten von Gesellschaften typisieren lassen etwa in demokratische, autoritäre und totalitäre mit jeweils einigen Subtypen (Tenscher und Viehrig 2010, 16ff) -, finden sich deren allgemeine Normen, Strategien und Maßnahmen auch als spezielle im Umgang mit dem Netz: In demokratisch-marktwirtschaftlichen Gesellschaften dürfte das Internet zusammen mit anderen Faktoren die weitere Expansion der Märkte, also die fortschreitende Einvernahme bislang nicht ökonomisierter zivilgesellschaftlich oder öffentlich verfasster Bereiche etwa durch soziale Netzwerke forcieren - eine Tendenz, die bereits als Ökonomisierung und/oder Kommerzialisierung skizziert wurde. In autoritären Regimen komplettiert und perfektioniert es die institutionelle Überwachung und Kontrolle innerhalb der nationalen Grenzen. Da es aber prinzipiell grenzüberschreitend und subversiv nutzbar ist, trotz aller rigider Zensurund Überwachsungsapparaturen, verhilft es auch nonkonformistischen und oppositionellen Gruppierungen zur Kommunikation und Publikation und befördert so deren gesellschaftliche Potentiale - mindestens insoweit, dass sie vor der Weltöffentlichkeit nicht mehr verheimlicht werden können. Außerdem lässt sich keine Bevölkerung mehr total von den international kursierenden Informationen und Bilder abschotten und nur mit den erlaubten, konformistischen Ideologien abspeisen; kollektive Bewusstseinsmanipulation und totalitäre Gehirnwäsche funktionieren mithin nicht mehr uneingeschränkt, zumal wenn auch die weitere Steigerung des Bildungsniveaus wirtschaftlich erforderlich ist. Allerdings scheinen überschwängliche Hoffnungen auf die positiven Kräfte des Internets zu verstummen: $\mathrm{Ob}$ und inwieweit die künftige digitale Information und Kommunikation via internationale Netze für die überkommenen Strukturen und Machtverteilungen in den Nationalstaaten, für die Erosion von Ideologien und die Zuspitzung oder Abschwächung von Konflikten positive oder negative Wirkung entfaltet, dürfte sich jedenfalls nicht in absehbarer Zeit entscheiden. Auch die noch jüngst als Handy- und Internet-Revolutionen gelobten Umwälzungen in den arabischen Ländern sind mittlerweile wieder in Bahnen traditioneller Auseinandersetzungen zurückgekehrt und haben ihre ursprüngliche Dynamik weitgehend eingebüßt (Steinschaden 2012).

Eine schleichende Umwälzung scheint die soziale Dimension - mindestens in den entwickelten Gesellschaften - durch Internet und digitale Mobilgeräte erfahren zu haben, allen voran durch Handy und heute Smartphones: Soziale 
Netzwerke - wie die Plattformen inzwischen euphemistisch heißen - bestreiten einen Großteil der davor noch unmittelbar, persönlich, „lebensweltlich“ (Habermas 1982, II, 229ff) und nichtkommerziell getätigten Beziehungen und Interaktionen (Ebersbach u.a. 2008; Emmer u.a. 2011): Soziale Kontakte, Gruppenbildung, Identitätskonstrukte, Selbstdarstellungen und Fremdpräsentationen, Diskussionen, Partnersuche, Sehnsüchte und Phantasievorstellen über das Zusammenleben, viele sonstige Verrichtungen des Alltags, aber auch Firmenwerbung, Unternehmenskommunikation, Bewerbungen, gewerbliche Informationen werden mittlerweile über die sozialen Netzwerke - allen voran Facebook - abgewickelt, verdrängen und ersetzen überkommene Kommunikationsformen, schaffen sicherlich neue Optionen, aber auch infolge ihrer tendenziell allen verfügbaren und nicht individuell mehr beeinflussbaren Zugänglichkeit neue Risiken (Meyen und Jaff-Rüdiger 2009; Weissensteiner und Leiner 2011). Denn all diese Systeme haben - schon systemlogisch bedingt - die mächtige Tendenz, immer mehr Funktionen und Aufgaben sich einzuverleiben und die Kunden an sich zu binden. Unter soziologischer Prämisse werden vorrangig Nutzungsprozesse, ihre Muster, Motive und ihre soziale Verteilung untersucht, wie sie unter dem Schlagwort „digital divide“ angesprochen sind. Allerdings ist eine wirksame, digitale Sozialpolitik noch kaum entwickelt; allein ein noch unzureichender Verbraucherschutz kümmert sich um die Gefahren möglicher Manipulation und Betrügerei. Und auch die Bildungspolitik besitzt umfassende, zeitgemäße Konzepte für die ,Internet-Generation', allenfalls in Modellversuchen und Experimenten werden sie erprobt (Kammerl u.a. 2011; Deutscher Bundestag 2011b; Dittler und Hoyer 2012).

Vorderhand transformieren die Entwicklungen des Internets die überkommene mediale Kommunikation und die etablierten, professionellen Medien. Aus kommunikationstheoretischer Sicht lässt sich eine Verschmelzung (Konvergenz) der durch die technischen Medienentwicklung seit der Erfindung des Buchdrucks entstandene Trennung von personaler und Massenkommunikation diagnostizieren, allerdings eine, die weitgehend auf digitaler Technik beruht. Dadurch werden technische Produktions- und Verbreitungsoptionen tendenziell für alle zugänglich und verfügbar, die publizistischen Professionen de-professionalisieren sich und Laien-Journalismus breitet sich aus, wie an Twitter, Blogs, Foren, Websites und anderen Hobby-Produkten erkenntlich wird. Doch die digitalen Medien ergänzen nicht nur die überkommenen, sie verdrängen sie auch zusehends, wie es besonders der gesamte Printbereich inzwischen zu spüren bekommt (Ruß-Mohl 2009; Neuberger und Quandt 2010). Prognostizierte man anfangs jene Konvergenz, also die Verschmelzung ,alter' und ,neuer' Medien unter digitalen Hybrid-Konstruktionen, zeichnen sich inzwischen eher Absorptionsprozesse ab, die - wenn sie gelingen - essentielle Geschäftsfelder (wie etwa Annoncen, 
Werbung, Public Relations, aber auch Spiele und Unterhaltung) ins Netz verlagern und die herkömmlichen Geschäftsmodelle beeinträchtigen. Auch bei aller demonstrativ gezeigten Aufgeschlossenheit der etablierten Anbieter: Bislang reüssieren kaum kombinierte Angebote und Einnahmestrukturen. Noch wird bei Buch und Print, bei Radio, Kino und Fernsehen die größere Akzeptanz und der höhere Umsatz mit den traditionellen Formaten - die selbstverständlich in Produktion und Verbreitung schon partiell digitalisiert sind - erzielt, während die neuen digitalen Offerten bislang noch eher Nischenstatus haben und quersubventioniert werden müssen.

Denn auch die Nutzungsgewohnheiten prägen die weitere Entwicklung (Schweiger 2010; Engel und Mai 2010; van Eimeren und Frees 2012): Zwar hat die Internetnutzung zumal der jüngeren Bevölkerungsgruppen zwischenzeitlich ein beachtliches Maß erreicht und differenziert sich - je nach den diversifizierten Angeboten - weiter aus, aber die klassischen Massenmedien sind noch nicht abgeschrieben, jedoch erfahren Presse und Buch erhebliche Einbußen. Allerdings müssen für ihre Nutzung größerer Aufwand und auch höhere, über Bildung vermittelte Kompetenzen aufgebracht werden, so dass Mediennutzung nach wie vor sozial differenziert ist. Da alle Medien weitgehend um die Befriedigung ähnlicher Motive buhlen, mindestens was Unterhaltung, Entspannung und Spiel angeht, müssen die Anbieter, um Aufmerksamkeit, Akzeptanz und damit Einkünfte zu erzielen, verstärkte Anstrengungen in Eigenwerbung, Attraktivität und Publikumsorientierung stecken. Vieles ist zwar präsent und publik, wird aber immer weniger wahrgenommen und rezipiert: Rezipienten und User werden mithin fortwährend umworben oder auch vereinnahmt, was die vorgebliche freie Wahl berührt. Je nach Bildung und Medienkompetenz werden die versierten Nutzer ihre bevorzugten Angebote finden, auch in den Nischen des Angebots, während das eher unbedarfte Publikum bei den lauten und schrillen Mainstream-Offerten hängenbleibt. Somit könnten sich neue Divergenzen oder Spaltungen auftun, die sich entlang der überkommenen Bildungs- und Kulturschranken ziehen.

Auch Nutzungsmodalitäten ändern sich, was vielfach schon an dem sich verbreitende Habitus des Surfens, des alinearen Link-Hoppings und der Ex-und-HoppMentalität kulturkritisch angemerkt wird: Häufige Internet-User seien nicht mehr fähig, einer längeren, ruhigen Textfolge oder Aufführung zu folgen, sondern übertragen ihre Hektik und Ungeduld auf Lektüre, Film und Fernsehen. Hinzukommt die simultane Nutzung mehrerer Medien, von Lektüre und Fernsehen, von Musik und Computer, Fernsehen und Internet. Die versierten Anbieter reagieren auf und stimulieren diese Nutzungsmultiplikationen mit immer ebenfalls multicodal aufgerüsteten Offerten: zusätzliche Laufbänder und eingeblendete Zusatzbilder im 
herkömmlichen Fernsehen, weitere begleitende Informationen und Hinweise über Links im digitalen TV, vielfach auch zu Werbezwecken genutzt. Außerdem kann die individualisierte Adressierung durch IP(Internet-Protokoll)-basierte Cookies forciert werden, wie auch das Nutzungsverhalten durch solch personalisierte Messung präziser erhoben wird. Schließlich dürfte auch die Fernsehnutzung dem allgemeinen Trend der Mobilisierung folgen: Auf Displays von Smartphones, iPads und Tablet-PC wird unterwegs fern- und/oder vedio gesehen, allerdings mit geringeren Qualitätsansprüchen und ganz kurzen Sequenzen, worauf sich die Bilderproduktion schon einstellt. Nur zu Hause, auf einem überdimensionierten Bildschirm, werden Spielfilme in HD- und 3D-Qualität genossen.

\subsection{Einige konkrete Entwicklungen}

Formuliert man zusammenfassend zentrale Dimensionen, so lässt sich konstatieren: In einer relativ kurzen Zeitspanne ist das Internet zu einem wesentlichen Bestandteil der globalen Wirtschaft geworden. Weltweit nutzten es $2011 \mathrm{ca}$. zwei Milliarden Menschen, beruflich und privat (Margetts 2009; OECD 2011), mit steigender Tendenz an Anzahl der User und Nutzungszeiten. Die Internet-Wirtschaft kommt schon für einen beachtlichen Teil der Wirtschaftsleistungen in den Industriestaaten auf (mehr als 8 Prozent), mehr noch zum Wachstum von Dienstleistungen und Beschäftigung. In einigen Industriestaaten ist der Wachstumsbeitrag der Investitionen in die Kommunikationsnetze bereits ebenso groß wie der aller übrigen Investitionen, das gleiche gilt für das Wachstum der Arbeitsproduktivität. Schließlich konzentriert sich ein wachsender Anteil der Forschungsausgaben und der erlangten Patente auf die Kommunikationstechnologien (OECD 2011b). Gleichzeitig hat diese spektakuläre Entwicklung und wirtschaftliche Bedeutungszunahme neue Befürchtungen hinsichtlich der Verlässlichkeit, Sicherheit und Offenheit des Internetverkehrs genährt (OECD 2008). Wenn die Vernetzung der Produzenten, Lieferanten und Kunden von der Sicherheit des Netzes abhängt, verursachen Störungen natürlich entsprechenden Schaden.

Trotz seines erheblichen Wachstums stecken das Internet und seine Nutzung aber eher noch in den Kinderschuhen: Nur ein Teil der weltweiten Bevölkerung - etwa ein Drittel - hat es überhaupt jemals genutzt, der größere Teil (über 80 Prozent) davon wiederum nicht regelmäßig (CISCO und GBN 2010). Nur ein kleiner Teil der weltweiten wirtschaftlichen Transaktionen findet digital statt, davon entfällt wiederum nur ein Bruchteil auf den Austausch zwischen Einzelhändlern und ihren Kunden. 
Ausgehend von diesen bereits existenten Dimensionen zeichnen sich folgende weitere Entwicklungen ab, die gleichwohl angesichts der bereits beschriebenen Trends und Prognoseprobleme nur als weitgehend heuristische zu begreifen sind:

1. Der internationale Wettbewerb zwischen Wirtschaftsstandorten wird sich erheblich intensivieren; dabei wird es auch gerade darum gehen, Anteile am globalen Datenverkehr, der Datenbearbeitung und der internet-basierten Wirtschaft zu ergattern. Zudem wird sich, wenn sich die Dynamik dieser Regionen so fortsetzt, der weltwirtschaftliche Schwerpunkt voraussichtlich stark in den asiatischen Raum und in andere Schwellenländer verlagern. Sie weisen im Durchschnitt eine deutlich jüngere Bevölkerung und eine wachsende, zunehmend konsumstarken Mittelschicht auf. Sie verfügen zudem über beträchtliche Wachstumsreserven, da bislang dort ein noch relativ hoher Anteil der Beschäftigten in der Landwirtschaft und in anderen, weniger produktiven Sektoren beschäftigt ist. Die Verstädterungsrate ist ebenfalls noch vergleichsweise niedrig. Mit dieser wirtschaftlichen Schwerpunktverlagerung wird sich das Nutzerprofil im Internet stärker zu diesen Ländern bzw. Gruppen verlagern, während die Nutzer in den alten Industriestaaten nur noch begrenzt zunehmen werden. Nach Schätzungen von CISCO und GBN (2010) wird die Hälfte des Internet-Verkehrs 2025 in den sogenannten aufstrebenden Volkswirtschaften stattfinden. Nach einer neuen Schätzung des Entwicklungsprogramms der Vereinten Nationen ist schon heute die Zahl der Internet-Nutzer in Entwicklungs- und Schwellenländern genau so groß wie in den alten Industrieländern (UNDP 2013).

Diese etablierten Industriestaaten, am stärksten die Mitgliedsländer der Europäischen Union, haben daher erhebliche Sorge, den Anschluss an die neue Zeit zu verlieren. So führt die Europäische Kommission aus, dass der Sektor der Informations- und Kommunikations-Technologien (IKT) gesamtwirtschaftlich zunehmend an Gewicht gewinnt, jedoch noch stärker zur gesamtwirtschaftlichen Produktivitätssteigerung beiträgt - Schätzungen reichen bis zu 50 Prozent - und zur Änderung der Lebensstile (Europäische Kommission 2010a). Im Wettrennen um einen größeren Anteil an den rasch expandierenden digitalen Märkten sieht die Kommission Europa bislang nicht sonderlich gut positioniert: Andernorts (genannt werden China, Korea und die USA) haben sich Hochgeschwindigkeits-Glasfasernetze viel rascher verbreitet als in Europa; es gibt dort große, einheitliche, nicht durch nationale Flickenteppiche geprägte Märkte und eine effizientere und breitere Nutzung der Online-Dienste. Außerdem wird deutlich mehr in zukunftsorientierte IKT-Forschung investiert (Ebd. und European Commission 2012d). Die digitalen Dienste in Europa arbeiten nicht so gut zusammen, wie es 
möglich wäre. $\mathrm{Zu}$ wenig wird in digitale Forschung und Entwicklung investiert. Vor allem existiert ein gravierender Mangel an IKT-Fachkräften trotz starker und steigender Nachfrage. Kurzum, die Kommission hat die zur Behebung der genannten Mängel entworfene Digitale Agenda für Europa zu eine der sieben Leitinitiativen der Strategie Europa 2020 erhoben, mit der die Mitgliedsländer für den globalen Wettbewerb fit gemacht werden sollen. Über die Erfolgschancen der europäischen Strategie braucht nicht weiter spekuliert zu werden; klar ist aber, dass die Konkurrenz nicht schläft - entsprechende strategische Entwürfe gibt es auch in den USA und bei sämtlichen Schwellenländern - und sich der digitale Wettbewerb verschärfen wird.

2. Die Zahl der „digital natives“, also derjenigen, für die das Internet zu einem alltäglichen Kommunikationsmedium geworden ist, wird deutlich zunehmen, möglicherweise auch ihr Umgang mit vergleichsweise anonymen Kontaktpartnern im Netz und mit persönlicher Information. Im Maße, wie diese "natives“ in Unternehmen einrücken, werden sich diese auf netzbasierte Geschäftskommunikation, Vertrieb, Forschung, Zahlungsvorgänge (etwa mobile Geldbörsen) verlassen. Viele Dinge des täglichen Lebens sollen künftig intelligent vernetzt sein. Im Internet der Dinge sollen Handys, Autos, Kühlschränke und Fahrkartenautomaten miteinander kommunizieren. Die Nutzer können sich dabei zunehmend einer breiteren Palette von mobilen, internetfähigen Geräten bedienen, die alle miteinander kommunizieren. Mobilgeräte sollen ihren Besitzern künftig abgespeicherte Artikel oder Bücher vorlesen können, künftige iPods werden die Musik direkt aus dem Internet laden und abspielen. Die Techniken dazu sind schon weitgehend entwickelt. Die Nutzung wird freilich von verfügbaren Breitbandnetzen, der Fähigkeit der Geräte und Standards zur Interoperabilität und - last not least - von ausreichenden finanziellen Mitteln zum Erwerb zusammenhängen. Eine digitale Kluft wird vermutlich bestehen bleiben - freilich in ganz unterschiedlichen kulturellen und sozialen Ausprägungen. Flächendeckend werden Technologien für die elektronische Identität und die Authentifizierung (andere als Passwörter) zum Einsatz kommen, der Verkehr mit den öffentlichen Behörden wird weitestgehend über das Netz abgewickelt werden. Die bisher üblichen Zugangswege zum Netz (über das Keyboard) werden vermutlich durch eine Kombination von Stimm-, Gestenund Bioerkennungsfazilitäten ersetzt. Das wird den Zugang für neue, auch technisch relativ unbedarfte Nutzergruppen erleichtern.

Intelligente Suchmaschinen (Web 3.0) brauchen einheitliche, aber offene Standards für die Beschreibung und Verknüpfung von Informationen und für die Kommunikation in natürlicher Sprache. An diesen wird bereits intensiv gearbeitet (Schibrowski 2011). Das „semantische Netz“ bietet Lösungen für die 
unüberschaubare Datenfülle im Netz, etwa die Integration von Informationen aus verschiedensten Quellen. Ein aktuelles Beispiel ist Travel IQ, ein Dienst, der auf Reise-Webseiten alle verfügbaren Flüge, Hotels und Mietwagen zusammensucht und sie nach den Bedürfnissen des Anfragers sortiert. Maschinen werden künftig vollautomatisch Nachrichten und Börsenreports schreiben können. Ein entsprechendes Programm für Sportnachrichten (der US-amerikanischen Firma Statsheet) arbeitet schon (Die Welt vom 2.5.2012). Klassische Suchmaschinen könnten dadurch überflüssig werden, selbst Google kann nicht so viel Manpower bereitstellen, „who would help to create those ontologies for the large set of knowledge domains that Wikipedia covers". Allerdings bleiben Fragen offen wie: Wer entscheidet, welche Informationen bzw. Verknüpfungen für den Endbenutzer relevant sind? Wie unterscheidet man selbst dann noch zwischen guter und schlechter Information? Und sie stellen sich erneut, wenn so genannte künstlicher Intelligenz weitere Aktivitäten übernimmt.

3. Da die meisten Institutionen - sind sie erst einmal eingeführt und haben sich einen Anhang (von Produzenten/Nutzern) geschaffen, für die Änderungen Kosten bzw. Verluste bringen - ist es nicht sehr wahrscheinlich, dass sich am bisherigen Modell der Internet Governance schnell und entscheidend etwas ändert, was nicht ausschließt, dass es - bedingt durch Hacker-Angriffe und CyberKriminalität - nicht immer wieder Bestrebungen zu einer stärkeren staatlichen Kontrolle des Netzes geben wird. Staaten beanspruchen geographische Namen und die Repräsentation ihrer Sprachen im Netz; auch sind sie bestrebt, das Netz (offensiv) zur Abwehr von Sicherheitsgefährdungen zu nutzen oder Inhalte zu filtern und zu zensieren. Damit lassen sich Cyber-Kriminalität bekämpfen und die Vertragsdurchsetzung in der digitalen Wirtschaft gewährleisten. Die gesellschaftliche Selbstorganisation im Netz findet darin ihre Schranken. Genauso unwahrscheinlich ist es aber auch, dass die künftige Internet Governance gänzlich $\mathrm{zu}$ einer durch staatliche Grenzen und staatliche Instanzen bestimmten Struktur zurückkehren wird (Mueller 2010). Voraussichtlich wird es mehr oder weniger beim bestehenden ,Multi-Stakeholder'-Ansatz von Internet Governance bleiben - ein zugegebenermaßen recht unpräzises Konzept. Staaten werden aber nicht Gleiche unter Gleichen sein (wollen), sondern durch ihre Machtressourcen, wirtschaftlichen Ressourcen und ihren Einfluss auf internationale Institutionen die dominanten Akteure bleiben.

4. Mit der Zunahme der Breitbandnutzung werden voraussichtlich neue Nutzerpreismodelle Einzug halten werden, welche die verfügbare Netzkapazität zeit- und nutzerbezogen stärker staffeln werden. Bisher spielt die differenzierte Preisgestaltung beim Zugang zu Daten noch kaum eine Rolle; dies wird sich mit 
Sicherheit ändern. Aber schnellerer und verlässlicher Zugang wird tendenziell mehr kosten (CISCO und GBN 2010)).

5. Mit der weiteren Optimierung und Expansion des Internets werden sich die Optionen zur Kontrolle, zur Filterung und Blockierung von Datenströmen verfeinern und vertiefen. Eine neue Technologie (Deep packet inspection) ermöglicht die umfassende und vor allem rasche Überwachung und Differenzierung der durch das Internet geschickten Datenpakete. Bei flächendeckender Anwendung könnten Internet Service Provider (ISP) und natürlich auch staatliche Stellen den ihnen nun bekannten Datenverkehr ihrer Nutzer prüfen, verlangsamen oder beschleunigen, filtern oder blockieren. ISP könnten damit auch viel stärker als bislang für den Transport illegaler bzw. unerwünschter Daten verantwortlich gemacht werden. Das hätte umfangreiche Auswirkungen auf den Datenschutz und die Informationsfreiheit im Netz vorausgesetzt, der Einsatz dieser Technologie wird nicht durch die bisherigen Regularien beschränkt (Bendrath und Mueller 2011).

6. Immer billiger werdende digitale Datenspeicherung erlaubt vor allem autoritären Systemen die komplette Ausspähung ihrer Bürger, auch ihrer Vergangenheit. Das Netz macht zwar die Organisation politischer Rebellionen einfacher, die Unterdrückung der daran Beteiligten aber eben auch. So wird es bei vertretbaren Kosten für solche Regime künftig möglich sein, die Aufenthaltsorte von all ihren Bürgern (über Mobiltelefone, GPSEmpfänger) minutenschnell zu identifizieren, ihre sämtlichen Anrufe, SMS und E-Mails jahrelang zu speichern und das alles miteinander abzugleichen. Die Begeisterung über die politische Ermächtigung von Dissidenten durch das Netz könnte also rasch umschlagen (Villasenor 2011).

7. Die Verbreitung des Internets und die damit erfolgende Verfügbarkeit von Nachrichten, Werbung, TV-Sendungen usw. haben bekanntlich ältere Medien bereits in Mitleidenschaft gezogen - eine Entwicklung, die sich zweifelsohne weiter verstärken wird. In vielen Industrie-, auch schon in manchen Schwellenländern befinden sich die Zahl der Tageszeitungen, ihre Auflage, Redaktionsstärke, Leserschaft und Anzeigeneinnahmen bereits seit wenigen Jahren im Sinkflug (insbesondere in den USA und Großbritannien). Insolvenzen von Tageszeitungen häufen sich (OECD 2010). Vor allem jüngere Nutzer (Spitzenreiter ist die Gruppe der 25- bis 34-jährigen) informieren sich zunehmend durch meist noch kostenfreie, aktualitätsorientierte Online-Medien. Die Frage stellt sich natürlich dann, ob mit dem Absterben alter Medien nicht zwangsläufig ein journalistischer Qualitätsverlust einhergehen wird, zumal die Online-Quellen aus der Verbreitung ihrer Nachrichten bislang nur bescheidene Einnahmen generieren. Umgekehrt sind Tageszeitungen durch schrumpfende Abonnentenzahl 
umso mehr auf Anzeigenerlöse angewiesen, was ihre Unabhängigkeit weiter untergraben könnte. Ihr Rekurs auf weniger und schlechter ausgebildete Mitarbeiter beeinträchtigt die Qualität der Berichterstattung, ein wesentliches Element informierter, demokratischer Gesellschaften. Klar ist damit, dass neue und alte Anbieter von Nachrichten neue Geschäftsmodelle für ausreichende ertragsstarke und verlässliche Berichterstattung brauchen.

\subsection{Einige Maßnahmen künftiger Internet Governance}

Bereits 2011 wurden unter Wissenschaftlern weltweit Debatten und Konzepte für ein neues Internet ventiliert, das nicht mehr allein der Maxime „Einfach weiter so" folgt (Fischermann und Hamann 2011a). Folgende „Forderungen ans nächste Netz“ (Ebd.) sind aufgestellt worden; realisiert sind sie bislang nicht:

1. Die Überladung und Überlastung des (einen) Netzes muss gestoppt werden; der Datenverkehr im Netz muss differenziert werden, oder - besser noch mehrere Netze für diverse Daten müssen geschaffen werden: „Kraftwerke und Stromumspannanlagen, Verkehrsleitsysteme und lebenswichtige Computer in Krankenhäusern, Flughafeneinsatzzentralen und alle möglichen Arten großer, schwerer Gerätschaften und Industrieanlagen“ (Ebd.) - sie alle haben Zugang zum Internet und wickeln darüber ihre Aktivitäten ab. Dadurch entstehen erhebliche Gefahren und Risiken, die nur mittels spezieller, interner Netze mit besonderen Sicherheitsstandards eingedämmt werden können und so für Missbrauch nicht ,knackbar‘ sind. Solche sensiblen Bereiche müssen vom Internet getrennt werden, ebenso wie die persönlichen Daten jedes Einzelnen besser geschützt werden sollten.

2. Bei Datenverlust und Vergehen gegen den Datenschutz sollte das Verursacherprinzip ähnlich dem Umweltrecht eingeführt werden. Unternehmen müssen für Nachlässigkeiten und Schäden haftbar gemacht und mit pauschalen, hohen Schadenssummen belegt werden können. Dadurch werden ökonomische Anreize für höhere Vorsicht und konsequentere Schadensabwehr geschaffen. Voraussetzung dafür sind absolute Transparenz und eine internationale Gerichtsbarkeit, bei der die Kunden ihren Verlust oder Schaden einklagen können.

3. Die ständige Anhäufung persönlicher Daten muss reduziert und ihre dadurch nicht mehr gewährleistete Sicherheit gestärkt werden. Persönliche Daten sollten nur dort erfragt und erhoben werden, wo es unbedingt erforderlich ist; bei 
anderen Online-Tätigkeiten wie Shopping genügen weit weniger gefährliche Angaben: Statt „Identifikation“ nur „Authentifizierung“ der Akteure, wobei sie sich nur mit einem Zahlencode „ausweisen“ müssen.

4. Daten sollten dort gespeichert werden, wo sie entstehen. „Dann kann jeweils die nationale Gesetzgebung [- in Europa auch die europäische -] greifen, dann gibt es die Grundlage dafür, dass rechtsstaatliche Aufsicht wirklich durch die jeweiligen Nationalstaaten [- oder internationalen Instanzen -] ausgeübt wird“ (Ebd.). $\mathrm{Ob}$ diese Maxime noch für die transnationale und -kulturelle Beschaffenheit angemessen und zeitgemäß ist, darf allerdings bezweifelt werden.

Für die nationale, also deutsche Situation ist schon mehrfach von Experten eine erhöhte Beachtung und vor allem Koordination der einschlägigen (Netz- und Medien-)-Politiken gefordert worden, die bislang noch in Rundfunk-, Medien-. und Telekommunikationsrecht aufgeteilt, sowie am besten ihre Bündelung in einer Instanz (Schiphorst u. a. 2012): „Die Medienpolitik gehört wieder in die erste Reihe der politischen Prioritäten und nicht in Expertenrunden hinter verschlossen Türen. Die vernachlässigten Aufgaben der digitalen Gesellschaft müssen entschlossen angegangen werden [...]“ (Ebd.).

Im Einzelnen werden folgende Initiativen und Maßnahmen gefordert (Holznagel und Schumacher 2012):

1. Für den Cyberspace müssen grenzübergreifende Grundwerte geschaffen und propagiert werden: Da infolge des grenzüberschreitenden Charakters des Internets rechtliche Fragen und Regulierungen nicht ausschließlich oder gar vorrangig in nationaler Reichweite geregelt und durchgesetzt werden können, müssen bestehende Grundwerte erweitert oder neue geschaffen werden, um die erforderlichen technischen, sozialen und kulturellen Spezifika des Netzes und die daraus resultierende Veränderungen in die internationalen Menschrechtserklärungen, die regionalen und nationalen Verfassungen aufzunehmen und ihnen möglichst hohe Gültigkeit und Nachdrücklichkeit zu verschaffen.

2. Auch für die Rechte des geistigen Eigentums und der Urheber, für Patent- und Kopierschutz sollten möglichst weltweite Regelungen und Abkommen angestrebt werden. Der unlängst gescheiterte Vorstoß (ACTA, vgl. Abschn. 6.6) belegt den weiterhin bestehenden Bedarf für weniger inkompetent eingebrachte Vorlagen.

3. Der Schutz der persönlichen Daten kann nicht nur als statisches Präventionsund Abwehrgesetz gefasst werden, sondern muss jüngere Entwicklungen auch im Bewusstsein der User einbeziehen, damit er überhaupt befolgt wird. Bei 
ihnen lässt sich eine verbreitete „Kultur des Teilens“ beobachten. Deshalb sollen künftig im Datenschutz auch ein „Recht auf Vergessen“ sowie ein „Recht auf Datenmitnahme“, um das Entstehen konkurrierender Internetangebote zu fördern, einbezogen werden, wie sie die gegenwärtig diskutierte neue Datenschutzrichtlinie der EU impliziert.

4. Der geltende Jugendmedienschutz-Staatsvertrag (JMStV) wird einem zeitgemäßen Jugendmedienschutz im Informationszeitalter kaum mehr gerecht. Marktbeschränkungen und Sendezeitreglungen sind an traditionelle MedienDistributionen geknüpft, die das Netz längst überwunden hat, und zeugen auch von einem überholten Nutzungsverhalten und Nutzerbild. Doch für einen zeitgemäßen Jugendmedienschutz engagieren sich keine politischen Kräfte und Initiativen.

5. Hinsichtlich der Netzneutralität scheut sich der deutsche Gesetzgeber, Anforderungen zu fixieren, die den Zugang zu Inhalten und Anwendungen diskriminierungsfrei sicherstellen. Dadurch ist bislang auch kein Ausgleich zwischen den Ansprüchen internationaler Telekommunikationskonzernen, den Interessen von Rundfunkanbietern, Telefonfirmen und der zivilgesellschaftlichen Netzgemeinden zu erreichen.

6. Für die wachsende gewerbliche, politische wie private Nutzung des Netzes und den schnellen Zugriff auf Internetdienste bedarf es des zügigen und konsequenten Ausbaus einer möglichst angemessenen und flächendeckenden Breitbandversorgung mit Glaserfaserkabel. Die Bundesregierung hat dazu ambitionierte Ziele formuliert, deren Realisierung, also Finanzierung und Bau, sie allerdings den Telekommunikationsunternehmen aufbürden will, so dass die geplante Versorgung in der vorgesehenen Zeit nicht mehr erreicht werden kann.

7. Die politisch zwischen Bund, Ländern und Kommunen aufgeteilten Kompetenzen in der Medien- und Netzpolitik verhindern deren strategische Ausrichtung und Wahrnehmung in allen, hier genannten Handlungsfeldern. Die besonderen Ansätze der EU intervenieren zusätzlich in diese Konzepte, zumal die EU im Datenschutz, Urheberrecht und Netzausbau übergeordnete Kompetenz reklamiert. So entscheiden vielfach die Märkte und involvierten Unternehmen, so dass demokratische Willensbildung und Kontrolle gerade bei der für alle Sektoren wichtigen Netzpolitik vielfach ausgehebelt sind. In Summe weist dies darauf hin, dass Internet Governance zunehmend weniger Sache und Kompetenz der gewählten und legitimierten Instanzen in Land, Bund und Europa sind, sondern oftmals künftig wohl kaum weniger - unauffällig und unkontrolliert von den Märkten und den maßgeblichen wirtschaftlichen Kräften vollzogen werden. 